\title{
Prostatic Artery Embolization in the Treatment of Benign Prostatic Hyperplasia: Short and Medium Follow-up
}

\author{
Hugo Rio Tinto, MD, ${ }^{*}$ João Martins Pisco, MD, PhD, ${ }^{*}$ Tiago Bilhim, MD, PhD,* \\ Marisa Duarte, MD, ${ }^{\dagger}$ Lúcia Fernandes, MD, ${ }^{\dagger}$ José Pereira, $\mathrm{MD},{ }^{\dagger}$ and \\ L. Campos Pinheiro, MD, $\mathrm{PhD}^{\ddagger}$
}

\begin{abstract}
To evaluate the short and mid-term results of prostatic artery embolization in patients with benign prostatic embolization. Retrospective study between March 2009 and June 2011 with 103 patients (mean age 66.8 years, 50-85) that met our inclusion criteria with symptomatic benign prostatic hyperplasia. The clinical outcome was evaluated by the International Prostate Symptom Score (IPSS), quality of life (QoL), International Index of Erectile Function, prostate volume (PV), prostate-specific antigen (PSA), peak urinary flow $\left(Q_{\max }\right)$, and post-void residual volume (PVR) measurements at 3 and 6 months, 1 year, 18 months, and 2 years after PAE and comparison with baseline values was made. Technical and clinical successes, as well as poor clinical outcome definitions, were previously defined. In this review, we evaluate the short and mid-term clinical outcomes and morbidity of patients treated only with non-spherical polyvinyl alcohol. Six months after the procedure, the PV decreased about 23\%, IPSS changed to a mean value of 11.95 (almost 50\% reduction), the QoL improved slightly more than 2 points, the $Q_{\max }$ changed to a mean value of $12.63 \mathrm{~mL} / \mathrm{s}$, the PVR underwent a change of almost half of the baseline value, and the PSA decreased about $2.3 \mathrm{ng} / \mathrm{mL}$. In the mid-term follow-up and comparing to the baseline values, we still assisted to a reduction in PV, IPSS, QoL, PVR, and PSA, and an increase in $Q_{\max }$. Prostatic Artery Embolization is a safe procedure with low morbidity that shows good short- and mid-term clinical outcome in our institution. Tech Vasc Interventional Rad 15:290-293 (c) 2012 Elsevier Inc. All rights reserved.
\end{abstract}

KEYWORDS prostate artery, embolization, benign prostatic hyperplasia

B enign prostatic hyperplasia (BPH) has a prevalence of over $50 \%$ in men older than 60 years. ${ }^{1}$ It is associated with lower urinary tract symptoms such as nocturia, urgency and leaking, hesitant, interrupted, and weak urinary stream, and in some patients, sexual dysfunction. ${ }^{2,3}$

Treatment depends on disease staging. Medical therapy is usually the first line. ${ }^{4,5}$ Transurethral resection of the prostate is the standard treatment. However, it can be

*Hospital de Saint Louis, Interventional Radiology and Universidade Nova de Lisboa Medical School, Department of Radiology, Lisbon, Portugal.

$\uparrow$ Hospital de Saint Louis, Interventional Radiology, Lisbon, Spain.

\#Hospital de São José, Urology Department and Universidade Nova de Lisboa Medical School, Lisbon, Portugal.

Address reprint requests to: João Martins Pisco, MD, PhD, Department of Interventional Radiology, Saint Louis Hospital, Rua Luz Soriano, no. 182, 1200-249 Lisbon, Portugal. E-mail: jpisco@hslouis.pt performed only with prostates smaller than $60-80 \mathrm{~cm}^{3}$, and is associated with some complications. ${ }^{6-8}$ Prostatic artery embolization (PAE) has been suggested as a safe and effective alternative procedure, reducing clinical symptoms and associated with reduction in PV. ${ }^{9-11}$

\section{Materials and Methods}

This was a retrospective study between March 2009 and June 2011 of patients aged 50-85 years (mean 66.8 years old) that met our inclusion criteria. Our inclusion criteria were male patients older than 50 , with symptomatic BPH refractory to medical treatment for at least 6 months, with International Prostate Symptom Score (IPSS) $>18$ points and/or Quality of Life (QoL) $>3$ and/or peak urinary flow $\left(Q_{\max }\right)<12 \mathrm{~mL} / \mathrm{s}$ and/or with acute urinary retention. Baseline PV was $>40 \mathrm{~mL}$ with sexual dysfunction or 
accepting the risk of developing it after embolization. Exclusion criteria were malignancy, evaluated by prostatic digital examination, prostate-specific antigen (PSA), transrectal ultrasound, and biopsy in cases with clinical or imaging suspicion of malignancy. We also excluded patients with severe atherosclerosis and tortuosity either in the aortic bifurcation or internal iliac arteries evaluated by computed tomographic angiography by our team of interventional radiologists prior to the embolization and as part of our preembolization workup. Patients with detrusor failure, neurogenic bladder, urethral stenosis or bladder diverticulum, or stone with surgical indication were also excluded.

The clinical outcome was evaluated by IPSS, QoL, International Index of Erectile Function (IIEF), PV, PSA, $Q_{\max }$ and postvoiding residual volume (PVR) measurements at 3 months, 6 months, 1 year, 18 months and 2 years after PAE. We considered poor outcome after PAE when 1 criteria was met: IPSS $\geq 20$ or reduction $<25 \%$ or both, QoL $\geq 4$ or reduction $<1$ or both, $Q_{\max }$ improvement $<2.5 \mathrm{~mL} / \mathrm{s}$ and additional treatments required, which were either medical or surgical.

All the patients were embolized using the same interventional technique by the femoral approach, in which the large majority was embolized with unilateral femoral approach. Bilateral approach was used in patients with significant atherosclerosis and tortuosity. The end-point was slow flow or near stasis with interruption of arterial flow and prostatic opacification. A subjective pain score (0-10) was used to assess pain during, immediately after, and 6-8 hours after the procedure. A score of zero means no pain and 10 means the worst pain felt. During the procedure we also registered the total procedure time as well as the fluoroscopy time.

We considered complications in the clinical situations that resulted from non-target embolization (related to the specific procedure) or those general situations that are possible after any interventional procedure (related to any interventional procedure). We did not consider complication situations such as postembolization syndrome, mild pain, or small hematomas at the puncture site. Exceptions were made in situations in which the patient required hospitalization or readmission. Situations were considered to be minor complications if they were easily managed ambulatorily and major if they led to prolonged hospitalization or readmission. The patients were evaluated during the day of the procedure before the discharge, the day after, and weekly for the first month. At that time we evaluated the puncture site, clinical signs of possible ischemic or infectious complications, and the patient's pain. Our follow-up protocol included physical, imaging, and laboratory evaluations. In this paper we evaluate the short- and mid-term clinical outcomes and morbidity of patients treated only with non-spherical polyvinyl alcohol in concentration of $100 \mu \mathrm{m}$ or $200 \mu \mathrm{m}$, or both.

\section{Results}

Between March 2009 and June 2011 we included 103 patients older than 50 years with indication of PAE considering our criteria. We achieved technical success in 100 patients ( 7 unilateral and 93 bilateral embolization). In the remaining 3 patients, the procedure was not possible due to extreme tortuosity and atherosclerosis of the aortic bifurcation and internal iliac arteries or prostatic artery origins that made selective catheterization impossible. Three patients had had a prostatic resection intervention previously.

During the procedure each patient's pain ranged from 0 to 10 (mean 1.6). Eighty-two patients did not feel any pain. Only 1 patient felt pain scored at level 10. After the procedure this was the only patient who experienced pain with the medication given according to our medication protocol.

Sixteen patients had a urinary catheter placed at the time of evaluation of preembolization due to a history of urinary retention. All of these patients had their catheter successfully removed in a maximum time period of 20 days after the procedure. All of the patients had spontaneously urinated with no recurrence of urinary retention in the short follow-up period. At 18 months follow-up there were 3 patients with urinary retention. In 2 of them we re-embolized the prostatic arterial supply bilaterally and the catheter was removed. The third patient had a prostatectomy.

The procedure times ranged from 25 to 185 minutes (mean time 83 minutes) and the fluoroscopy times were 7-63 minutes (mean time 24 minutes).

Ninety patients were discharged who did not show any symptoms during the same day of the procedure and the remaining group stayed 1 night following the procedure. During the first week of follow-up evaluation we observed 7 patients with inguinal hematomas. Fourteen patients had macroscopic hematuria and 6 had hematospermia. These last 2 situations were self-limited and did not require any specific treatment or intervention besides oral hydration. Sixteen patients developed urinary tract infection confirmed in microbiology examinations and they were treated with the most suitable antibiotics. Among these patients, the majority had a urinary catheter prior to the procedure.

Six patients had transient urinary retention, of which 2 of them required a temporary urinary catheter. Two patients had balanitis which could be easily managed with treatment with anti-inflammatory medications according to our postprocedure protocol. We reported 1 major complication, which was a bladder wall ischemia that led to an intraluminal necrotic tissue that was removed surgically and did not require bladder wall reconstruction. This patient had pain scored 10 in the pain-subjective evaluation during the procedure.

In the short-term follow-up we have 100 patients at 1 month, 83 patients at 3 months and 62 patients at 6 months. Mid-term follow-up included 29 patients at 1 year, 13 patients at 18 months, and 6 patients at 2 years.

Table 1 shows the mean values of PV, IPSS, QoL, $2_{\max }$, PVR, and PSA, and their range values.

At 3-month follow-up we had 13 patients with clinical failure. Some of them did not have any clinical 
Table 1 Baseline, Mean Values and Range of Evaluated Clinical Parameters

\begin{tabular}{|c|c|c|c|c|c|c|c|}
\hline & Baseline & 1 Month & 3 Months & 6 Months & 12 Months & 18 Months & 24 Months \\
\hline PV Mean (mL) & 88.0 & 68.7 & 67.0 & 67.8 & 68.0 & 72.5 & 76.0 \\
\hline (Min-Max) & $40-269$ & $25-183$ & $19-175$ & 21-170 & $21-170$ & $26-220$ & 26-127 \\
\hline IPSS Mean & 22.8 & 11.73 & 10.29 & 10.85 & 11.22 & 10.3 & 9.3 \\
\hline (Min-Max) & 4-35 & $1-31$ & $1-26$ & $0-29$ & $0-32$ & $0-27$ & $1-20$ \\
\hline \multirow{2}{*}{$\begin{array}{l}\text { QoL Mean } \\
\text { (Min-Max) }\end{array}$} & 4.11 & 2.26 & 2.04 & 1.96 & 2.01 & 2.0 & 2.0 \\
\hline & $2-6$ & $0-6$ & $0-5$ & $0-5$ & $0-5$ & $0-4$ & $0-4$ \\
\hline \multirow{2}{*}{$\begin{array}{l}Q_{\max } \text { Mean }(\mathrm{mL} / \mathrm{s}) \\
\text { (Min-Max) }\end{array}$} & 8.7 & 11.6 & 12.68 & 12.63 & 12.9 & 13.73 & 14.42 \\
\hline & $1.5-22.9$ & $2.8-30$ & $2.8-30$ & $3.9-29$ & $4-27.5$ & $4-42$ & 4.7-27.6 \\
\hline \multirow{2}{*}{$\begin{array}{l}\text { PVR Mean }(m L) \\
\text { (Min-Max) }\end{array}$} & 104.9 & 70.38 & 60.85 & 61.1 & 53.38 & 77.5 & 93.54 \\
\hline & $0-439$ & $0-361$ & $0-360$ & $0-361$ & $0-226$ & $12-280$ & $0-439$ \\
\hline \multirow{2}{*}{$\begin{array}{l}\text { PSA Mean (ng/mL) } \\
\text { (Min-Max) }\end{array}$} & 6.4 & 4.52 & 4.04 & 4.71 & 5.24 & 5.53 & 6.24 \\
\hline & $0.25-39.37$ & $0.4-43.8$ & $0.35-26.04$ & $0.35-25.95$ & $0.46-26.79$ & $0.4-16$ & $0.92-14.4$ \\
\hline IIEF Mean & 18.47 & 19.61 & 20.07 & 20,20 & 19.76 & 20.41 & 17.1 \\
\hline (Min-Max) & $0-34$ & $0-33$ & $0-30$ & $0-30$ & $0-31$ & $0-30$ & $0-29$ \\
\hline
\end{tabular}

PV, prostatic volume; IPSS, International Prostate Symptom Score; QoL, quality of life; Qmax, peak urinary flow; PVR, post-void residual volume; PSA, prostate-specific antigen; IIEF, International Index of Erectile Function; Min, minimum value; Max, maximum value.

improvement immediately after PAE and at 1-month follow-up. All these patients had the same symptoms after PAE (IPSS was higher than 20 and QoL $\geq 4$ points). Among these clinical failures, 3 had significant prostatic reduction and 5 had unilateral embolization. Two weeks after the procedures, we repeated the procedure in 2 patients that had unilateral embolization with good clinical outcome. So, we reduced the number of clinical failures to 11 patients in short-term follow-up.

Six months after the procedure the PV decreased about $23 \%$, IPSS changed to a mean value of 11.95 (almost $50 \%$ reduction), the QoL improved slightly more than 2 points, the $Q_{\max }$ changed to a mean value of $12.63 \mathrm{~mL} / \mathrm{s}$, the PVR underwent a change of almost half of the baseline value, and the PSA decreased about $2.3 \mathrm{ng} / \mathrm{mL}$ (Tables 1 and 2). In the mid-term follow-up and comparing to the baseline values, we still assisted to a reduction in PV, IPSS, QoL, PVR, and PSA, and an increase in $\mathcal{Q}_{\max }$.

\section{Discussion}

Majority of patients showed a significant clinical improvement with no prostatic medication. In comparison with other treatments, our results seem to be better than those obtained with medical therapy in this group of patients. The change in IPSS is similar to other minimally invasive techniques such as transurethral microwave thermotherapy and transurethral needle ablation and not significantly different compared to those with transurethral resection of the prostate, although they seem to have poorer symptom-score improvements. ${ }^{12,13}$

Most clinical failures were evident at short-term follow-up. We retrospectively evaluated our clinical failures and in some cases both sides were well embolized, with no specific reason for those unsuccessful results. Patients should be informed about the possibility of clinical failure after a successful PAE. The procedure can be repeated in cases that are technically feasible, which would be a patient with poor clinical outcome who fulfills the inclusion criteria.

We consider that computed tomographic angiography is crucial for the selection process, and therefore, for the results after the procedure. In particular, patients with severe pelvic arterial occlusive disease or tortuosity can be excluded on the basis of this preprocedure imaging.

The clinical success cannot be predicted based on PV itself or with other isolated parameters. Prostates with same volume reduction can have different clinical outcomes. We think that the factors that explained the

Table 2 Change in \% or Absolute Values from the Baseline Values

\begin{tabular}{lcccccc}
\hline & 1 Month & 3 Months & 6 Months & 12 Months & 18 Months & 24 Months \\
\hline PV (\% Change) & -21.93 & -23.9 & -23.0 & -22.7 & -17.61 & -13.64 \\
IPSS (Change) & -11.07 & -12.51 & -11.95 & -11.58 & -12.5 & -13.5 \\
QoL (Change) & -1.85 & -2.07 & -2.15 & -2.10 & -2.11 & -2.4 \\
Qmax (\% Change) & +33.0 & +45.7 & +45.2 & +48.2 & +57.8 & -26.12 \\
PVR (\% Change) & -32.9 & -41.99 & -41.75 & -49.11 & -10.8 & -2.5 \\
PSA (\% Change) & -29.4 & -36.8 & -26.4 & -18.1 & -13.9 & +1.94 \\
IIEF (Change) & +1.14 & +1.6 & +1.73 & +1.29 & -37 \\
\hline
\end{tabular}


clinical success are not completely understood and further studies are needed, with more patients and multicenter studies.

The general improvement in IIEF may possibly be explained by the interruption of the prostatic medication that is normally associated with a deterioration in sexual function. Further study is necessary to understand this seemingly paradoxical result with PAE.

There are several limitations in this study: we were not able to measure IPSS, QoL, and $Q_{\max }$ in the patients with urinary catheter and so the changes in these parameters can only be evaluated in other patients; this is a retrospective study with the use of polyvinyl alcohol in concentration of $100 \mu \mathrm{m}$ or $200 \mu \mathrm{m}$ or both; and outcomes should be intrepreted considering that the patients stopped the previous BPHmedication prior to PAE.

PAE is a safe procedure with low morbidity that shows good short- and mid-term clinical outcomes in our institution with no sexual dysfunction.

\section{References}

1. Levy A, Samraj GP: Benign prostatic hyperplasia: When to "watch and wait", when and how to treat. Cleve Clin J Med 74:S15-S20, 2007 (suppl)

2. Rosen RC, Giuliano F, Carson CC: Sexual dysfunction and lower urinary tract symptoms (LUTS) associated with benign prostatic hyperplasia (BPH). Eur Urol 47:824-837, 2005

3. Eckhardt MD, Van Venrooij GE, Van Melick HH, et al: Prevalence and bothersomeness of lower urinary tract symptoms in benign prostatic hyperplasia and their impact on well-being. J Urol 166:563-568, 2001

4. Michel MC, Mehlburger L, Brussel HU, et al: Tamsulosin treatment of 19365 patients with lower urinary tract symptoms: Does comorbidity alter tolerability? J Urol 160:784-791, 1998

5. McConnell JD, Bruskewitz R, Walsh P, et al: The effect of the finasteride on the risk of acute urinary retention and the need for surgical treatment among men with benign prostatic hyperplasia. N Engl J Med 338:557-563, 1998

6. Varkarakis J, Bartsch G, Horninger W: Long-term morbidity and mortality of transurethral prostatectomy: A 10-year follow-up. Prostate 58:248-251, 2004

7. Baazeem A, Elhilali MN: Surgical management of benign prostatic hyperplasia: Current evidence. Nat Clin Pract Urol 5:540-549, 2008

8. Reich O, Gratzke C, Bachman A, et al: Morbidity, mortality and early outcome of transurethral resection of the prostate: A prospective multicenter evaluation of 10654 patients. J Urol 180:246-249, 2008

9. Sun F, Sánchez FM, Crisóstomo V, et al: Benign prostatic hyperplasia: Transcatheter arterial embolization as potential treatmentpreliminary study in pigs. Radiology 246:783-789, 2008

10. Jeon GS, Won JH, Lee BM, et al: The effect of transarterial prostate embolization in hormone-induced benign prostatic hyperplasia in dogs: A pilot study. J Vasc Interv Radiol 20:384-390, 2009

11. DeMeritt JS, Elmasri FF, Esposito MP, et al: Relief of benign prostatic hyperplasia-related bladder outlet obstruction after transarterial polyvinyl alcohol prostate embolization. J Vasc Inter Radiol 11:767-770, 2000

12. Madersbacher S, Marberger M: Is transurethral resection of the prostate still justified? Brit J Urol Int 83:227-237, 1999

13. May F, Hartung R: Surgical treatment of BPH: Technique and results. EAU Update Series 2:15-23, 2004 